\title{
Increased SARS-CoV-2 Testing Capacity with Pooled Saliva Samples
}

\author{
Anne E. Watkins, Eli P. Fenichel, Daniel M. Weinberger, Chantal B.F. Vogels, \\ Doug E. Brackney, Arnau Casanovas-Massana, Melissa Campbell, John Fournier, \\ Santos Bermejo, Rupak Datta, Charles S. Dela Cruz, Shelli F. Farhadian, Akiko Iwasaki, \\ Albert I. Ko, Nathan D. Grubaugh, ${ }^{1}$ Anne L. Wyllie, ${ }^{1}$ the Yale IMPACT Research Team ${ }^{2}$
}

We analyzed feasibility of pooling saliva samples for severe acute respiratory syndrome coronavirus 2 testing and found that sensitivity decreased according to pool size: 5 samples/pool, $7.4 \%$ reduction; 10 samples/pool, $11.1 \%$; and 20 samples/pool, $14.8 \%$. When virus prevalence is $>2.6 \%$, pools of 5 require fewer tests; when $<0.6 \%$, pools of 20 support screening strategies.

$\mathrm{L}$ imited laboratory capacity in the United States has hindered access to testing for severe acute respiratory syndrome coronavirus 2 (SARS-CoV-2) and has delayed results. To control outbreaks of coronavirus disease (COVID-19), testing capacity must be increased and maintained for the foreseeable future. One resource-saving, capacity-increasing approach is pooling samples, thereby testing multiple persons simultaneously. A negative result for the pool indicates that all samples were below the limit of detection, and a positive result for the pool requires individual retesting of all samples. Pooled testing has been widely proposed as a way to expand capacity for large-scale screening (1,2; C.M. Verdun, unpub data, https:// doi.org/10.1101/2020.04.30.20085290), a proactive strategy for early pathogen detection, primarily for persons who are not yet symptomatic.

Saliva is being used as a noninvasive source for SARS-CoV-2 testing $(3,4)$ yet can be more difficult to process than traditional swab-based samples (5).

Author affiliations: Yale School of Public Health, New Haven, Connecticut, USA (A.E. Watkins, D.M. Weinberger, C.B.F. Vogels,

A. Casanovas-Massana, A.I. Ko, N.D. Grubaugh, A.L. Wyllie); Yale School of the Environment, New Haven (E.P. Fenichel); Connecticut Agricultural Experiment Station, New Haven (D.E. Brackney); Yale University School of Medicine, New Haven (M. Campbell, J. Fournier, S. Bermejo, R. Datta, C.S. Dela Cruz, S.F. Farhadian, A. Iwasaki, A.I. Ko); Howard Hughes Medical Institute, New Haven (A. Iwasaki)

DOI: https://doi.org/10.3201/eid2704.204200
Given limited empirical evidence to properly inform projections of feasibility and cost-effectiveness of pooling, we explored the potential of pooling saliva to increase SARS-CoV-2 testing capacity.

\section{The Study}

Using saliva collected from COVID-19 inpatients and at-risk healthcare workers (5), we combined 1 SARS-CoV-2-positive sample (<38 PCR cycle threshold $\left[C_{t}\right]$ ) with SARS-CoV-2-negative saliva (Appendix, https://wwwnc.cdc.gov/EID/article/27/4/204200-App1.pdf) before RNA extraction in total pool sizes of 5 samples/ pool ( $n=23$ pools), $10(n=23)$, and $20(n=31)$. As pool size increased, detection sensitivity decreased independent of starting viral load (pool of 5, +2.2 cycle threshold $\left[\mathrm{C}_{\mathrm{t}}\right], 95 \%$ CI 1.4-3.0 $\mathrm{C}_{\mathrm{t}^{\prime}} 10$, $+3.1 \mathrm{C}_{\mathrm{t}^{\prime}}, 95 \%$ CI 2.3-4.0 C $\mathrm{t}^{\prime} 20,+3.6 \mathrm{C}_{\mathrm{t}^{\prime}} 95 \%$ CI 2.7-4.4 $\mathrm{C}_{\mathrm{t}}$ ) (Figure 1; Appendix).

By applying the regression coefficients $\left(C_{t}\right.$ increase) to the $C_{t}$ values from all SARS-CoV-2-positive saliva samples detected during our studies (6), we estimate that pool sizes will lead to detection sensitivities of $92.59 \%$ (95\% CI $88.89 \%-95.56 \%$ ) for pools of 5 samples, $88.89 \%$ (95\% CI $80.00 \%-91.85 \%)$ for pools of 10 , and $85.19 \%(95 \%$ CI $75.56 \%-91.11 \%)$ for pools of 20, relative to sensitivity of unpooled samples (Appendix Figure 1). This loss in sensitivity could be minimized through protocol modifications: increasing the volume of pooled samples tested $(400 \mu \mathrm{L}, \mathrm{n}=20$ pools of each size; Appendix Figure 2) and decreasing the elution volume.

On the basis of the calculated relative sensitivity loss resulting from pooling, we modeled the number of tests required (total of pooled and individual samples from positive pools tested) for a population of 10,000 with increasing SARS-CoV-2 prevalence

\footnotetext{
${ }^{1}$ These senior authors contributed equally to this article.

${ }^{2}$ Team members team are listed at the end of this article.
} 
(Figure 2, panel A). We estimate that for populations with prevalence $<0.6 \%$, pools of 20 require the fewest tests. However, for populations with prevalence $>2.6 \%$, our analyses suggest that pooling of 5 samples leads to the fewest tests. For populations with prevalence $>28.1 \%$, testing individual samples is more efficient than testing pools of any size. Thus, we suggest using an adaptive pooling strategy that accounts for SARS-CoV-2 prevalence for the population tested: as virus prevalence decreases, pool size can be increased, but as prevalence rises, pool size should be decreased.

Because sensitivity varies by pooling design (Figure 1), a different number of positive results will be detected for a given population with a given SARS$\mathrm{CoV}-2$ prevalence. As virus prevalence decreases, we estimate that cost savings of pooled testing will increase (Figure 2, panel B). For example, if SARS-CoV-2 prevalence for a 10,000 -person population was $0.5 \%$, then pooling by 20 would require only 1,318 tests, including retesting of all persons from test-positive pools. If tests cost US\$30 each, the savings would be $\$ 260,453$ relative to individual testing while still identifying $\approx 43$ of 50 infected persons. The savings will vary on a scale relative to test prices. Ultimately, the net benefits of pooled testing can continue to increase even as virus prevalence decreases with increased pool sizes, which is essential for ongoing screening.

\section{Conclusions}

The cost of SARS-CoV-2 testing can be prohibitive when positive samples are rarely found, presenting a major barrier to prolonged screening strategies. Pooling of samples can help overcome this barrier. Our model demonstrates that as local outbreaks fluctuate, adapting pool sizes will have resource-savings benefits.

The benefits of pooled testing will always be accompanied by decreased detection sensitivity. However, the lower overall number of tests required and the lower associated costs expands testing capacity, permitting more frequent testing, and testing persons more often mitigates the loss of sensitivity (7). By enabling broader testing, pooling has the potential to identify more infected persons than more limited (or no) individual testing. Infected persons can then be isolated from the population, thus reducing the probability of contact between a susceptible and an infectious person, ultimately reducing transmission. Given our findings, we urge the US Food and Drug Administration to develop new guidelines for pooledtesting approaches. Although the first Emergency Use

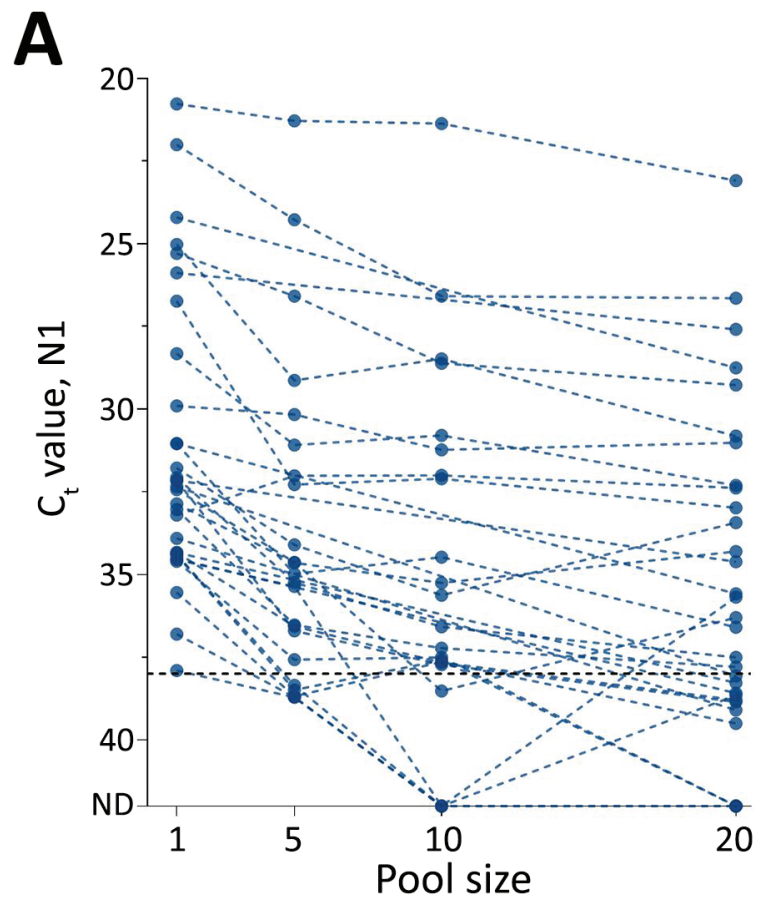

B

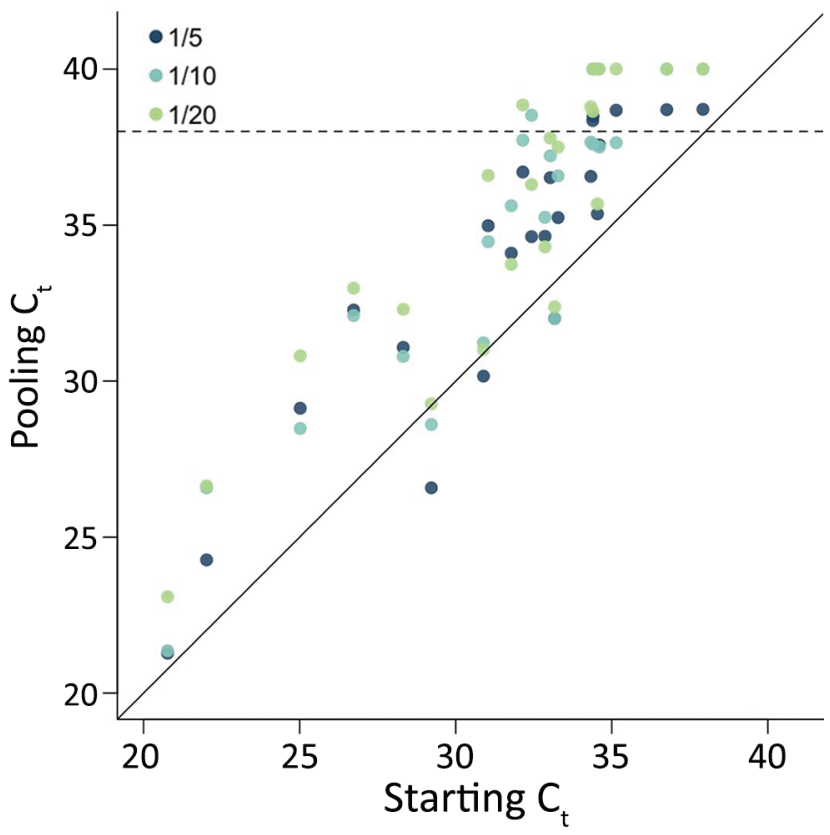

Figure 1. Effect of pooling on detection of severe acute respiratory syndrome coronavirus 2, by pool size and between samples tested. A) As the pool size increased, so did the $C_{t}$ value (dotted lines connect pools comprising the same positive sample). $C_{t}$ for positivity is set to 38. Samples falling on the $x$-axis indicated samples from which signal was not detected by reverse transcription quantitative $P C R$. B) As the pool size increased, so did the $C_{t}$. We equated this change by using linear regression (pool of 5 samples, dark blue, $+2.2 C_{t}$, $95 \% \mathrm{Cl} 1.4-3.0 \mathrm{C}_{\mathrm{t}}$; pool of 10 , light blue, $+3.1 \mathrm{C}_{\mathrm{t}}, 95 \% \mathrm{Cl} 2.3-4.0 \mathrm{C}_{\mathrm{t}}$; pool of 20 , green, $+3.6,95 \% \mathrm{Cl} 2.7-4.4 \mathrm{C}_{\mathrm{t}}$ ). Dashed lines indicate $C_{t} 38$ (cutoff for sample positivity). $1 / 5$, pool of $5 ; 1 / 10$, pool of $10 ; 1 / 20$, pool of 20 . $C_{t}$, cycle threshold. 

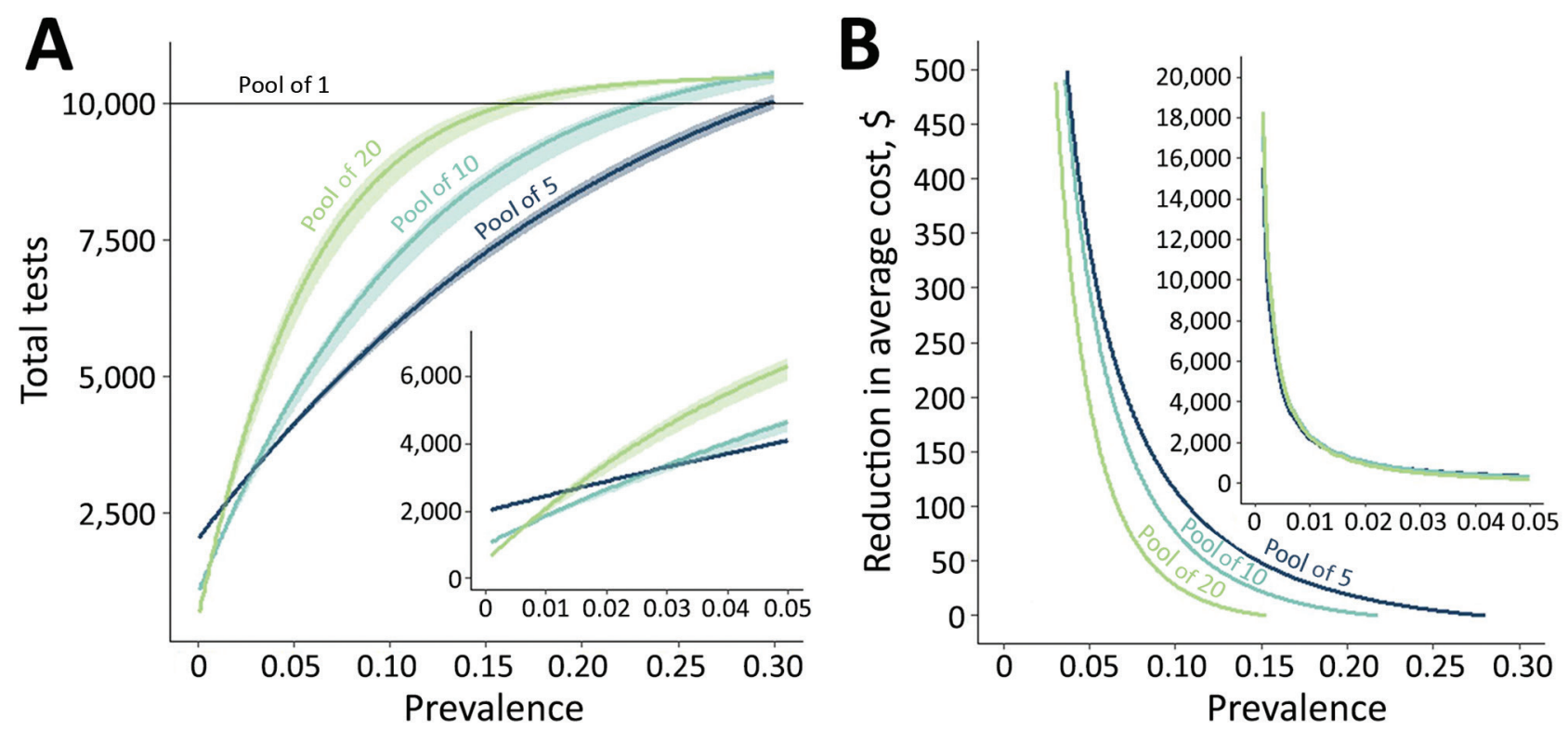

Figure 2. The resource-saving benefit of sample pooling for severe acute respiratory syndrome coronavirus 2 (SARS-CoV-2) testing, based on size of the pool and expected prevalence of SARS-CoV-2 within the population. A) We modeled the number of tests required to test 10,000 persons (results qualitatively scale with population) when pools contain 5,10 , or 20 samples (and individually retesting samples within positive pools) compared with testing samples individually (pool $=1$ sample). As prevalence increases, so does the number of pools positive for SARS-CoV-2, thereby increasing the required number of confirmatory tests of individual samples. Therefore, over a prevalence of $2.6 \%$, pooled samples of 5 result in fewer overall tests required than do larger pool sizes. B) At lower prevalence rates, such as when outbreaks have been controlled but ongoing screening is required, pools of 10 or 20 samples yield substantial cost savings for the same expected level of positive detections, after accounting for sensitivity differences. Values are shown in US\$. Insets show the region with $<5 \%$ prevalence.

Authorization for SARS-CoV-2 pooled testing ( $\leq 4$ swab samples in 1 test) (8) will be most useful in highprevalence settings, the $\approx 12 \%-15 \%$ losses in sensitivity when pooling 10-20 samples would probably not pass current authorization criteria ( $>95 \%$ sensitivity).

Going forward, screening strategies need to be reviewed separately from traditional diagnostic testing, taking into consideration the repeated testing of individuals performed during screening. For strategies considering twice-weekly sampling, such as in the reopening plans for many US colleges, even if larger pools have lower sensitivity per test, the probability of 2 repeated false-negative results for any person will often be less than the probability of a false-negative result for a single test from a small pool. For example, a small pool (or individual test) may have the probability of a false-negative result of $2 \%$ but cost may limit testing to once per week. Conversely, the lower per-person cost of a large pool with a per-test probability of a false-negative result of $14 \%$ is more likely to allow for testing twice per week. Therefore, persons tested twice in larger pools have a per-week false-negative probability of only $1.96 \%$. In the context of prolonged community screening, sensitivity should be thought of as per unit time, and the testing regimen should be taken into account.
Our estimates are conservative; the number of tests required is most likely lower than predicted, especially if behavioral or geographic information can be used to stratify the population so that the adaptive pooling strategy can be applied differentially to different sampled subpopulations. However, this approach needs to be balanced with feasibility in the laboratory because pooled testing adds additional steps and complexity to the system, all of which must be reliably implemented. Furthermore, pooled approaches could incorporate retesting individual samples from pools generating any SARS-CoV-2-specific signal in quantitative reverse transcription PCR regardless of $C_{t}$ (in place of those pools with the $<38 C_{t}$ cutoff applied here) (9). Although pooling has traditionally focused on extracted nucleic acid before quantitative reverse transcription PCR (10-12), because of the expense of RNA extraction and a comparable effect on detection sensitivity (Appendix), we recommend pooling before RNA extraction. Validation of our work in additional settings and on a larger scale will help better inform our models.

The cost-savings benefits of adaptive pooling saliva for community screening for SARS-CoV-2 provides a mechanism to maintain testing as virus spread 
is brought under control and to avoid resurgence. Even if prevalence is very low, it is probably desirable to increase pool sizes before stopping testing altogether. Together with the ease of saliva collection, pooling samples should be considered as an effective testing strategy for expanding the breadth of testing and continued screening during the ongoing COVID-19 pandemic.

Yale IMPACT Research Team authors: Kelly Anastasio Michael H. Askenase, Maria Batsu, Sean Bickerton, Kristina Brower, Molly L. Bucklin, Staci Cahill, Yiyun Cao, Edward Courchaine, Giuseppe DeIuliis, Rebecca Earnest, Bertie Geng, Ryan Handoko, Christina A. Harden, Chaney C. Kalinich, William Khoury-Hanold, Daniel Kim, Lynda Knaggs, Maxine Kuang, Eriko Kudo, Melissa Linehan, Peiwen Lu, Alice Lu-Culligan, Anjelica Martin, Irene Matos, David McDonald, Maksym Minasyan, Adam J. Moore, M. Catherine Muenker, Nida Naushad, Allison Nelson, Jessica Nouws, Abeer Obaid, Camila Odio, Ji Eun Oh, Saad Omer, Isabel M. Ott, Annsea Park, Hong-Jai Park, Xiaohua Peng, Mary Petrone, Sarah Prophet, Tyler Rice, Kadi-Ann Rose, Lorenzo Sewanan, Lokesh Sharma, Denise Shepard, Mikhail Smolgovsky, Nicole Sonnert, Yvette Strong, Codruta Todeasa, Maria Tokuyama, Jordan Valdez, Sofia Velazquez, Arvind Venkataraman, Pavithra Vijayakumar, Elizabeth B. White, and Yexin Yang.

\section{Acknowledgments}

We gratefully acknowledge the study participants for their time and commitment to the study. We thank all members of the clinical team at Yale-New Haven Hospital for their dedication and work, which made this study possible. We also thank S. Taylor and P. Jack for technical discussions.

This study was supported by the Huffman Family Donor Advised Fund (N.D.G.), Fast Grant funding support from the Emergent Ventures at the Mercatus Center, George Mason University (N.D.G.), the Yale Institute for Global Health (N.D.G.), and the Beatrice Kleinberg Neuwirth Fund (A.I.K.). C.B.F.V. is supported by NWO Rubicon 019.181EN.004.

\section{About the Author}

Ms. Watkins is a master's degree student and research assistant in Epidemiology of Microbial Diseases, Yale School of Public Health, in New Haven, CT. Her primary research interests are the intersection of infectious and chronic diseases with a focus on respiratory and neurologic pathogens.

\section{References}

1. Cherif A, Grobe N, Wang X, Kotanko P. Simulation of pool testing to identify patients with coronavirus disease 2019 under conditions of limited test availability. JAMA Netw Open. 2020;3:e2013075. https://doi.org/10.1001/ jamanetworkopen.2020.13075

2. Abdalhamid B, Bilder CR, McCutchen EL, Hinrichs SH, Koepsell SA, Iwen PC. Assessment of specimen pooling to conserve SARS CoV-2 testing resources. Am J Clin Pathol. 2020;153:715-8. https:/ / doi.org/10.1093/ajcp/aqaa064

3. Yokota I, Shane PY, Okada K, Unoki Y, Yang Y, Inao T, et al. Mass screening of asymptomatic persons for SARS-CoV-2 using saliva. Clin Infect Dis. 2020 Sep 25 [Epub ahead of print]. https://doi.org/10.1093/cid/ciaa1388

4. Vogels CBF, Brackney D, Wang J, Kalinich CC, Ott I, Kudo E, et al. SalivaDirect: simple and sensitive molecular diagnostic test for SARS-CoV-2 surveillance [cited 2021 Jan 27]. https:/ / www.cell.com/med/pdf/S2666-6340(20)30076-3.pdf

5 Landry ML, Criscuolo J, Peaper DR. Challenges in use of saliva for detection of SARS CoV-2 RNA in symptomatic outpatients. J Clin Virol. 2020;130:104567. https:/ / doi.org/ 10.1016/j.jcv.2020.104567

6. Wyllie AL, Fournier J, Casanovas-Massana A, Campbell M, Tokuyama M, Vijayakumar P, et al. Saliva or nasopharyngeal swab specimens for detection of SARS-CoV-2. N Engl J Med. 2020;383:1283-6. https://doi.org/10.1056/ NEJMc2016359

7. Larremore DB, Wilder B, Lester E, Shehata S, Burke JM, Hay JA, et al. Test sensitivity is secondary to frequency and turnaround time for COVID-19 surveillance. Sci Adv. 2021;7:eabd5393. https://doi.org/10.1126/sciadv.abd5393

8. Quest Diagnostics. Quest SARS-CoV-2 rRT-PCR package insert [cited 2020 Aug 20]. https:/ / www.fda.gov/ media/136231

9. Pasomsub E, Watcharananan SP, Watthanachockchai T, Rakmanee K, Tassaneetrithep B, Kiertiburanakul S, et al. Saliva sample pooling for the detection of SARS-CoV-2. J Med Virol. 2020 Aug 25 [Epub ahead of print]. https:// doi.org/10.1002/jmv.26460

10. Eis-Hübinger AM, Hönemann M, Wenzel JJ, Berger A, Widera M, Schmidt B, et al. Ad hoc laboratory-based surveillance of SARS-CoV-2 by real-time RT-PCR using minipools of RNA prepared from routine respiratory samples. J Clin Virol. 2020;127:104381. https:/ / doi.org/ 10.1016/j.jcv.2020.104381

11. Wyllie AL, Rümke LW, Arp K, Bosch AATM, Bruin JP, Rots NY, et al. Molecular surveillance on Streptococcus pneumoniae carriage in non-elderly adults; little evidence for pneumococcal circulation independent from the reservoir in children. Sci Rep. 2016;6:34888. https:/ / doi.org/10.1038/ srep34888

12. Yelin I, Aharony N, Tamar ES, Argoetti A, Messer E, Berenbaum D, et al. Evaluation of COVID-19 RT-qPCR test in multi-sample pools. Clin Infect Dis. 2020;71:2073-8. https:// doi.org/10.1101/2020.03.26.20039438

Address for correspondence: Anne L. Wyllie, Yale School of Public Health, 60 College St, New Haven, CT 06511, USA; email: anne.wyllie@yale.edu; Eli P. Fenichel, Kroon Hall, 195 Prospect St, New Haven, CT 06511, USA; email: eli.fenichel@yale.edu 